\title{
Use of Physical Models to Facilitate Transfer of Physics Learning to Understand Positron Emission Tomography ${ }^{*}$
}

\author{
Bijaya Aryal, Dean Zollman and N. Sanjay Rebello
}

Department of Physics, Kansas State University, Manhattan, KS, 66506-2601

\begin{abstract}
In this paper we describe a qualitative study of the role of the physical models in transferring physics ideas to understanding positron emission tomography technology. Sixteen students enrolled in an introductory level physics class individually participated in two sessions of a teaching experiment. In this study we noted that many students used reasoning from prior experiences in inappropriate ways. A result from this study is that physical models are effective in triggering appropriate transfer provided that the activities using the models are introduced in the right sequence. Given the appropriate sequencing of the activities, we find that the transfer of abstract ideas is facilitated through interactive learning with the aid of physical models. Three different types of non-scaffolded transfer have been identified: spontaneous, semi-spontaneous and non-spontaneous transfer.
\end{abstract}

Keywords: Physical models, positron emission tomography, transfer of learning

PACS: $01.40 \mathrm{Fk}$

\section{INTRODUCTION}

This study investigates the transfer of learning from ideas in physics to positron emission tomography (PET), a medical imaging device. Students cover some of the physics concepts relevant to PET such as momentum conservation and distance in introductory level physics. Thus, we investigate how students use those physics ideas in the context of PET. Rather than beginning with PET we first examine students' models of some of the physics ideas relevant to PET. Students learn from simple classical models and later apply those ideas to the electron-positron annihilation process in order to understand the image construction process in PET. For this purpose we conduct teaching interviews [1] to investigate not only students' models but also the ways in which we can facilitate student learning of the correct model.

Our aim in this study was to answer the following research questions.

1. What cognitive resources do introductory college students bring to bear when interacting with physical models?

2. How does sequencing of different physical models affect activation of these resources?

3. How do students transfer what they have learned in physics from physical models to their understanding of PET?

\section{LITERATURE REVIEW}

From the encoding specificity [2] perspective in cognitive psychology, transfer of learning is deemed easy if the problem structures are superficially similar in learning stage and transfer stage. Contrary to this view, some researchers [3] have reported that increasing concreteness of the problem does not promote transfer of abstract ideas.

We have developed activities to facilitate transfer of abstract ideas from one context to another. The activities in the learning and transfer contexts are therefore intended to be different at the superficial level but similar at an abstract level. For the learning session we designed structurally simple hands-on activities with underlying abstract physics concepts. On the other hand for the transfer session we introduced the problems that were similar to problems in the learning session at an abstract level but in different contexts and modes of presentation.

In this paper we mainly discuss the influence of students' prior ideas while learning PET's relevant physics principles using our interactive activities. Another part of discussion of this paper will be the transfer of learning from the models of the activities to the PET image construction process. 


\section{METHODOLOGY}

Sixteen students, eight females and eight males, participated in this study. The students were enrolled in an introductory algebra-based physics course. The study was done during the second half of spring 2006. We choose that time of the semester because students would be familiar with some basic kinematics terms by then.

The teaching interview sequence consisted of a fixed protocol with scaffolding activities introduced dependent upon students' responses. Each student participated in two different sessions, both one-hour in length, that were about a week apart. The sessions were conducted by one of the authors (BA) and some were observed by the other authors.

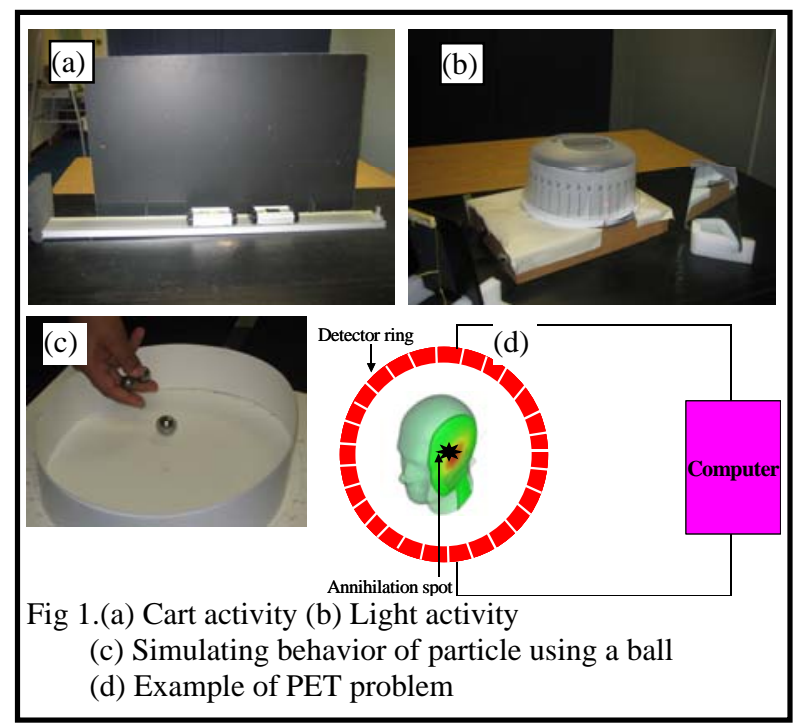

We introduced two activities in the first session. In both of the activities students were asked to figure out the locations of events that were not visible directly, but whose effects were visible. In the 'cart activity' in Fig. 1(a) two magnetic carts were brought together on a hidden track and then released. Students could see the carts only at the ends of the track. Given the velocity of the carts students were asked to determine the location on the track from where the carts were released. The 'light activity' in Fig. 1(b) simulated an explosion inside a cylindrical enclosure. The result was two pulses of light which were visible on the surface of the cylinder. Students could see two light pulses on the wall of barrier, but not the simulated explosion. To facilitate students' understanding of the particles that caused the simulated explosion in the 'light activity' a model of balls on a whiteboard was used (Fig 1 (c)). In this model, students were asked to predict the directions of two smaller balls resulting from the explosion of a bigger ball. Through these activities, students had to figure out the locations of such events and pinpoint the common region of such events.

The second session began with a discussion of general ideas about positron emission tomography (PET). We then moved on to problems related to the PET technique (See, Fig. 1(d)). Students were asked to build a model of how the location of the exact position of electron-positron annihilation in the brain could be ascertained. Finally they were asked to complete activities which enabled them to find the region of a tumor in which the annihilations were occurring.

The teaching activities of both sessions were video recorded and later transcribed. The field notes of the teaching interview during and after the activities and students' worksheets served as valuable data sources.

\section{RESULTS AND DISCUSSION}

A phenomenographic [4] analysis was conducted. We examined the activity transcripts, field notes and student worksheets to find recurrent categories. Different categories were labeled and their criteria were defined. An inter-rater reliability ranging from $72 \%$ to $84 \%$ was established among five researchers.

\section{Reasoning Resources}

The following themes emerged from the analysis:

\section{Central Tendency:}

Most of the students (87\%) located the events in both the cart and light activities at the center of a circle or line. For instance, in the light activity, students explicitly mentioned that if two lights appeared at two points on the circumference the source producing these lights must be at the center of that circle. When asked to explain their reasoning most students appeared to have arrived at this conclusion based on their intuition.

During the teaching interview we challenged this idea. Figure 2 shows a representative sketch of how students' ideas progressed with scaffolding.

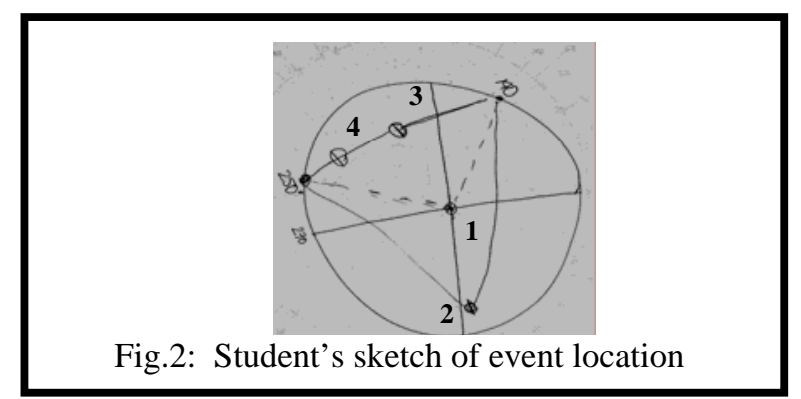

The stages are labeled as 1, 2, 3 and 4 in the sketch. Before receiving any hints the student thought that the event should be at the center (1) and later decided that it could be anywhere inside the circle (2). 
After the student was told that two fragments from an explosion produce light, he said that the event must be at the center of a line joining two lights (3). Finally he realized that it could be anywhere along that line (4). It is reasonable to deduce that a symmetry argument might have led them to have the central tendency.

\section{Factors to Predict Event Location Along a Line:}

About $40 \%$ of the students who participated in this study relied on intensity of light and another 20\% relied on size of light to locate the events. The appropriate factor to be considered was time, which was considered by $40 \%$ of the students. It is interesting to note that most of the students who considered 'time' were engaged in the cart activity before the light activity as discussed further in the next section.

\section{Predictions of Number and Direction of Gamma Rays:}

Most of the students used classical analogies and everyday experiences to explain the number and direction of gamma rays produced by annihilation (i.e. collision of automobiles and balls rolling on a table). A huge majority (87\%) of students stated that only an even number of gamma rays could cancel momentum to conserve it. The following statement by a student is a typical example of this line.

There have to be...almost...there have to be an even number... that way...so that for each one produced it may...it will have opposite one it will cancel out so that it doesn't have any movement anywhere...

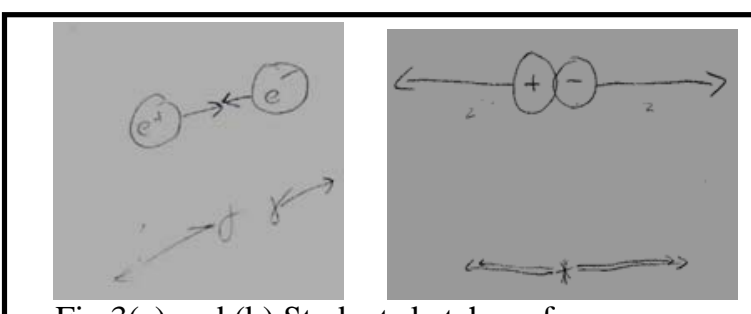

Fig 3(a) and (b) Student sketches of gamma ray direction as a result of annihilation

Figures 3(a) and 3(b) illustrate an idea held by a majority of the interviewees (73\%) regarding the direction of gamma rays. Many of them explicitly mentioned that a conservation law should be applied in direction also. They said that the conservation of the line should hold so that the gamma rays should travel back along the same line that the electron and positron traveled before they met to annihilate. Upon probing we found that this was a result of the influence of the 1-dimensional activities that they had seen in lecture demos and other prior activities.

\section{Sequencing Activities}

The two activities used in first session were introduced in two different sequences. For the first group (LC) of 11 students the light activity was introduced first followed by the cart activity. For the second group (CL) of five students the cart activity was introduced before the light activity. The second group had fewer participants because students were unavailable.

In response to a question on how to locate the event producing light in the light activity seven out of 11 from the LC group mentioned that light intensity should be the determining factor whereas two students mentioned the apparent size of the light. The remaining two students considered that the time for the light to reach the cylinder would be the determining factor. In the second (CL) group on the other hand, all five students considered 'time' to be the relevant factor to locate the event that produces light.

We speculate that most of the students in the second group (CL) activated their resource of 'time' as being associated with the 'location' of the event. This activation was apparently based on the cart activity. Shortly afterward they were introduced to the light activity. Apparently the associations they made between 'time' and 'location' with the cart activity were so strong that the light activity which followed was unable to suppress the activation of the 'time' resource and displace it with the 'intensity' or 'size' resource. But, for first group of students they didn't have an opportunity to make an association of 'time' with 'location' with the aid of cart activity before doing the light activity. So, they activated their resources from their everyday life experiences where they usually locate lights based on their intensity or size. This group of student appeared to have activated the p-prim 'closer is brighter' and 'closer is bigger' [5] to conclude that the location should be determined by the intensity or size of the lights. This p-prim-based association was apparently so strong that it could not be displaced by the association between 'time' and 'location' when the cart activity was introduced. In other words, the sequence of the activities CL vs. LC made a difference in the activation of knowledge resources by these students as they explained this activity.

\section{Transfer of Learning}

The main objective of this study was to see if and how students could transfer their learning from physical models to understand PET. Thus, in the first session we involved students with classical hands-on activities using physical models. The purpose of the second session was to help them learn the physics of PET. The problem structures in both sessions were the 
same in level of abstraction. We observed that students' responses indicated a transfer of ideas from the first to the second session. We classified the responses into four categories.

Response indicates Spontaneous Transfer (ST) If students immediately related PET with the activities of the first session we labeled this as spontaneous transfer (ST). For example

Interviewer: How will the PET machine be able to determine the exact location here?

Student: By the process that we went through last time... knowing the difference in time...knowing which gamma ray reach the sensor first...so if the gamma ray reaches this sensor first and the computer can figure out which point it is in between the two sensors...

Response indicates Semi-Spontaneous Transfer (SST) If students related PET back to the activities of the first session upon being asked the reason for their answer we called this semi spontaneous transfer (SST). For example:

Interviewer: What caused you to answer in that way? Student: It is like the cars last week...where the event took place ...since you can't time whenever this event took place (refers to the annihilation)...then you could say whichever detector goes first and time it when it goes off and then the time to reach ...

Response indicates Non-Spontaneous Transfer (NST) The third category is non-spontaneous transfer (NST). We categorized these students if they related PET with the first session only after being asked if they had seen a situation similar to PET somewhere before. E.g:

Interviewer: Any prior learning prompted at this point?

Student: Last week when we did the cake exercise (light activity) trying to figure out the source of light that kinda helped too...

\section{Response indicates No Transfer (NT)}

There were some instances in which very few of the students did not transfer at all from the first session to the second session. Here is an example.

Interviewer: (after introducing the picture of coincidence detection) How does the machine get the exact location of annihilation?

Student: I can tell it ...can tell about here (detector).... can't tell how far from here ...I can't tell how to get the exact location... because I never saw this machine and don't know how it works...

Almost one half of the participants (47\%) in this study were found to be in the SST category. The second largest population (27\%) exhibited ST transfer. Only $13 \%$ of students demonstrated NST transfer and another $13 \%$ of students' responses were in the NT category. Unlike many of the earlier studies [6] we found significant transfer from learning context (first session) to transfer context (second session). It could, of course, be argued that the students already had some ideas about the transfer context due to hints that they were in a two-part study. But it is encouraging that they made an association with the activities of the first session with those of the second session without any hints in spite of the different problem structure in the two sessions. The students were not told about the activities in the transfer session by those in the learning session but they themselves built or changed their ideas while interacting with the physical models. This result suggests that the exercise helped them construct ideas by active learning and eventually led them to apply in the transfer task.

\section{CONCLUSIONS}

The results of this study show that the introductory physics students who participated in this study rely on everyday experiences even when dealing with complex physics problems. They also appear to transfer their learning from familiar physics experiments to new situations. Despite the usefulness of such prior ideas and reasoning, in some cases these transfer processes may have an adverse effect on learning.

The analysis of the activities of two groups of students showed the importance of sequencing different activities. Based upon these results, we can suggest that the sequence of the activities has an important role in activating different conceptual resources. This result has important implications in designing teaching materials. Depending upon the ideas we want students to apply in a new situation, we can decide where and when an activity should be introduced to facilitate spontaneous transfer for a majority of students.

\section{REFERENCES}

1 P. V. Engelhardt, E. G. Corpuz, D. J. Ozimek et al., presented at the Physics Education Research Conference, 2003, Madison, WI, 2003 (unpublished).

2 R. H. Bruning, Cognitive Psychology and Instruction. (Pearson, Upper Saddle River, NJ, 2004).

3 R.L. Goldstone and Y. Sakamoto, Cognitive Psychology 46 (414-446) (2003).

4 F. Marton, Journal of Thought 21, 29 (1986).

5 A. DiSessa, in Constructivism in the computer age, edited by G. Forman and P.B. Pufall (Lawrence Erlbaum Associates, Hillsdale, NJ, 1988), pp. 49.

6 M. Wertheimer, Productive Thinking. (Harper \& Row, New York, NY, 1959). 